
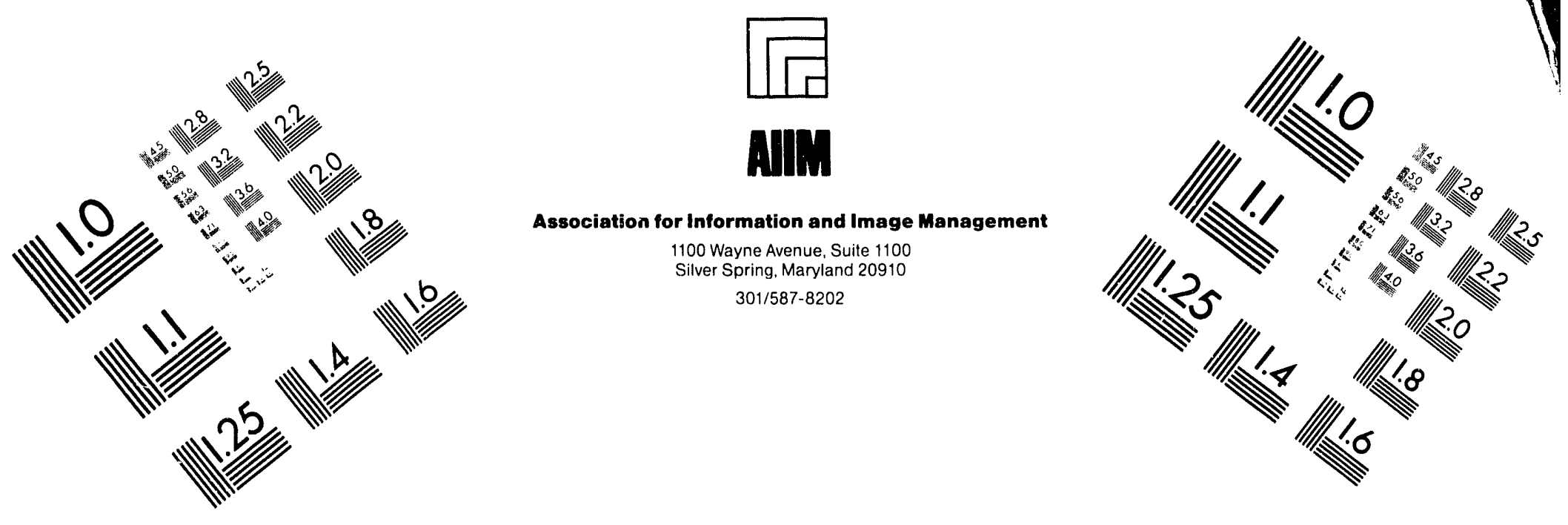

\title{
Centimeter
}

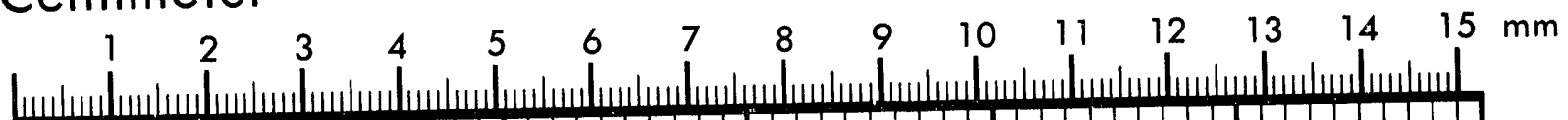

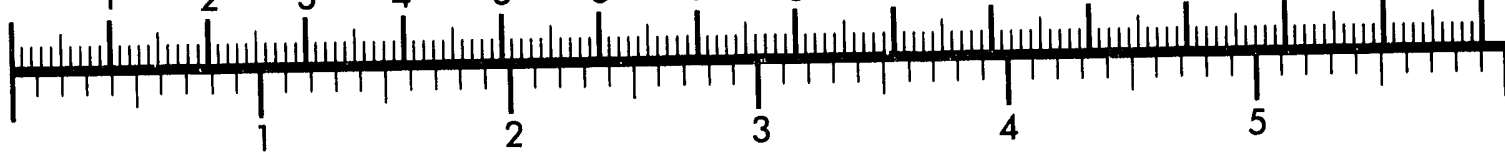
Inches
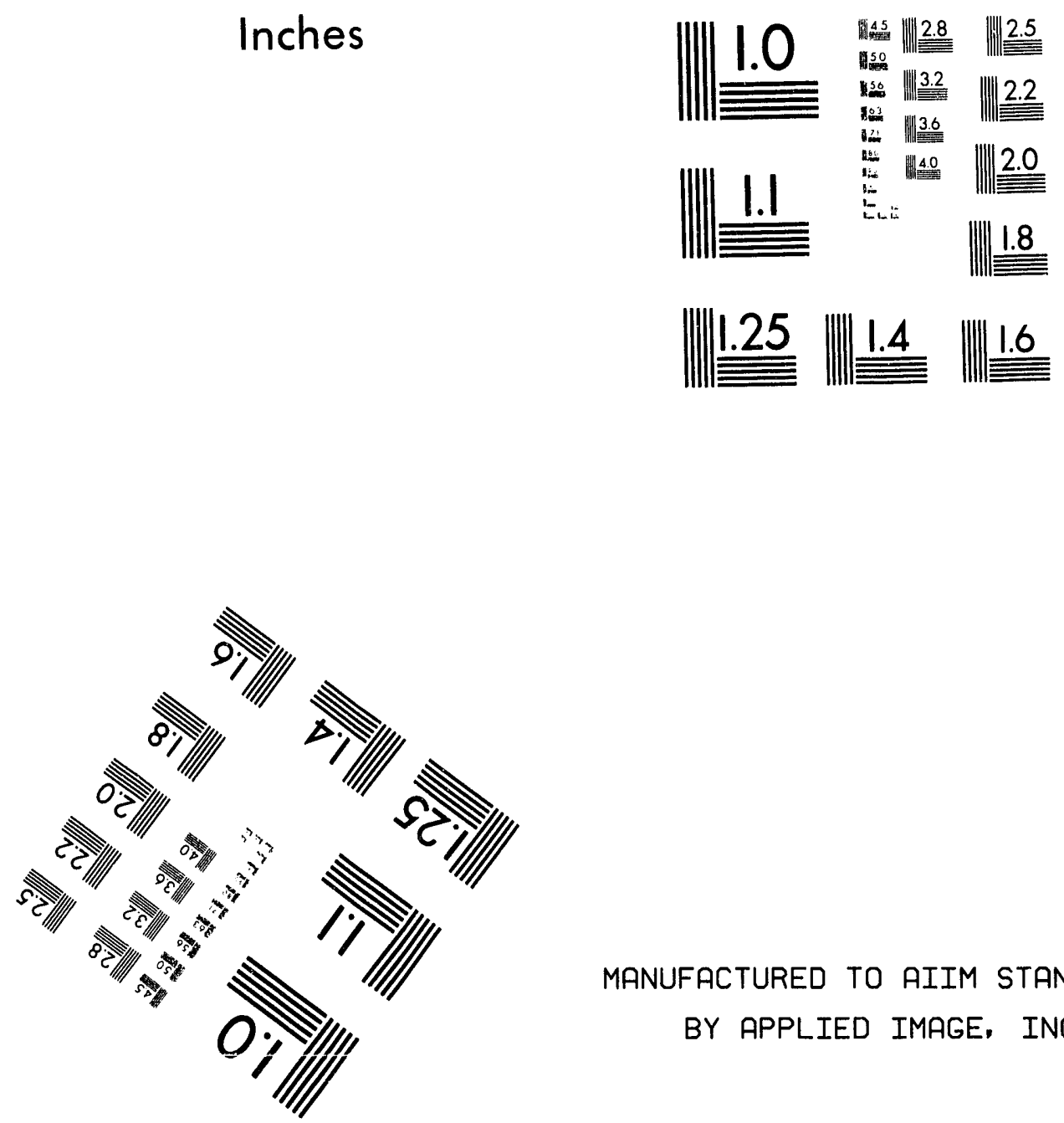

MANUFACTURED TO AIIM STANDARDS

BY APPLIED IMAGE, INC.

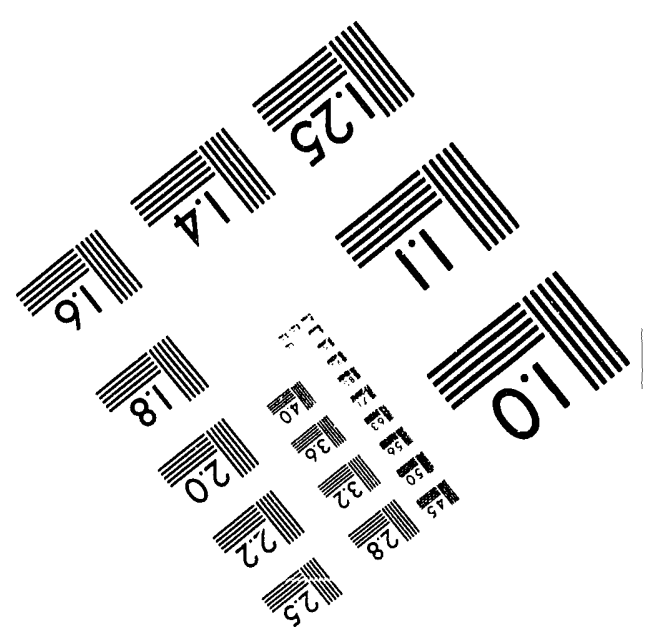



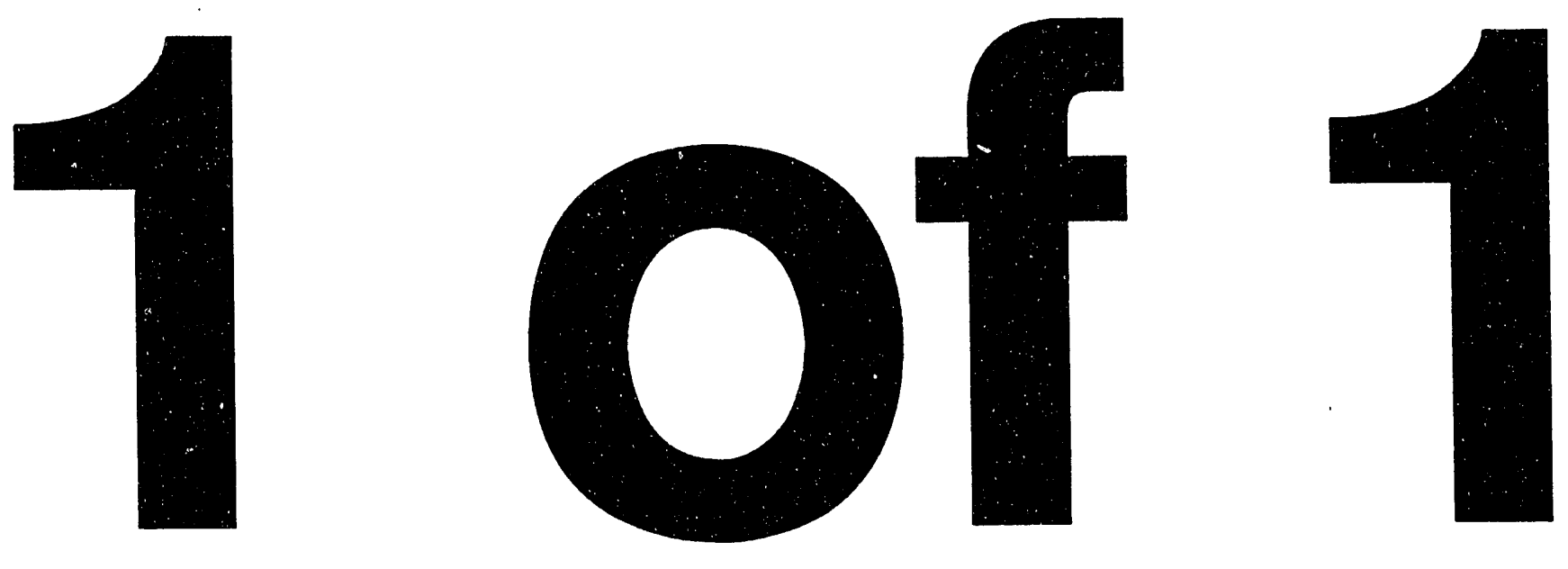
UCRL-JC-112438

PREPRINT

\section{Recent Studies of Short-Range Order in Alloys - The Cowley Theory Revisited}

L. Reinhard

S.C. Moss

This paper was prepared for submittal to the

Ultramicroscopy: Proceedings of the

John M. Cowley Symposium

Tempe, AZ

January 5-8, 1993

February 8, 1993

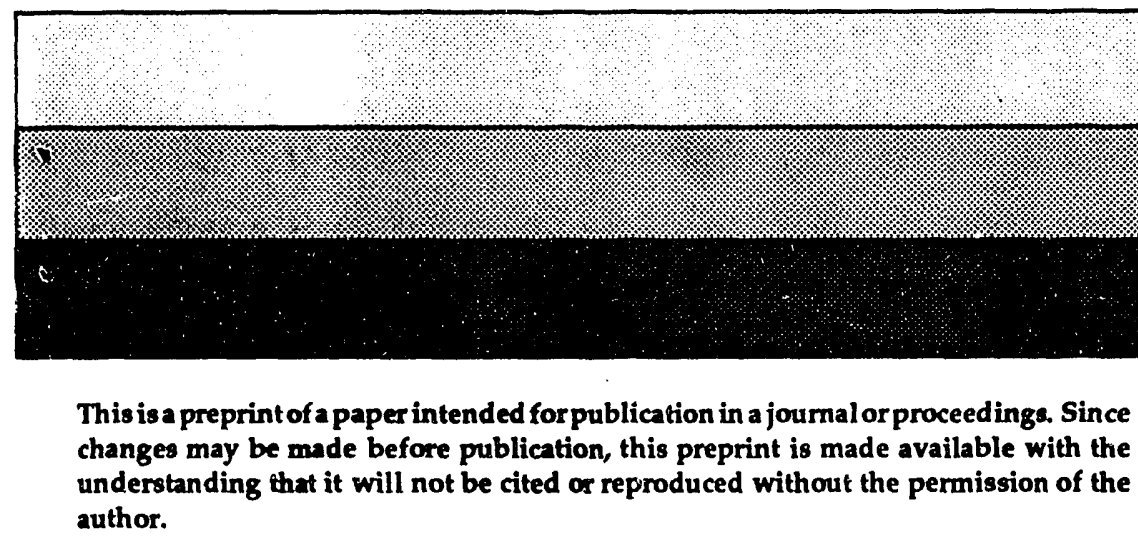

author. 
This document was prepared as an account of work sponsored by an agency of the United States Government. Neither the United States Government nor the University of California nor any of their employees, makes any warranty, express or implied, or assumes any legal liability or responsibility for the accuracy, completeness, or usefulness of any information, apparat us, product, or process disclosed, or represents that its use would not infringe privately owned rights. Reference herein to any specific commercial products, process, or service by trade name, trademark, manufacturer, or otherwise, does not necessarily constitute or imply its endorsement, recommendation, or favoring by the United States Government or the University of California The views and opinions of authors expressed herein do not necessarily state or reflect those of the United States Government or the University of Califomia, and shall not be used for advertising or product endorsement purposes. 
to appear in Ultramicroscopy:

Proceedings of the John M. Cowley Symposium, 5-8 January 1993

\title{
Recent Studies of Short-Range Order in Alloys - The Cowley Theory Revisited
}

\author{
L. Reinhard \\ Condensed Matter Division (L-268) \\ Lawrence Livermore National Laboratory \\ Livermore, CA 94550 \\ S. C. Moss \\ Physics Department, University of Houston \\ Houston, TX 77204-5506
}

\begin{abstract}
We present comparisons of various statistical theories for effective pair interactions (EPI) in alloys. We then evaluate these EPI's using the Cowley theory, the Krivoglaz-Clapp-Moss (KCM) approximation, the $\gamma$-expansion method (GEM) of Tokar, Masanskii and co-workers, and the exact inverse Monte Carlo (IMC) method, introduced by Gerold and Kern. Via a series of model calculations on a hypothetical bcc alloy with a single nearest-neighbor interaction we show that the Cowley theory is successful in evaluating the EPI's in more dilute alloys but tends to overestimate the magnitude of the nearest neighbor energy at higher concentrations, whereas the KCM expression becomes increasingly inaccurate at lower concentrations. In general, however, the approximate mean field theories are most accurate at higher concentrations and higher temperatures. Recent studies of short-range order in single crystals are discussed in which these EPI's have been evaluated using the IMC, KCM, GEM and Cowley theories. Examples include the bcc alloy $\mathrm{Fe}_{0.53} \mathrm{Cr}_{0.47}$ and the $\mathrm{fcc}$ alloys $\mathrm{Cu}_{3} \mathrm{Au}, \mathrm{Cu}_{0.69} \mathrm{Zn}_{0.31}$ and $\mathrm{Ni}_{0.89} \mathrm{Cr}_{0.11}$. In all cases the approximate expressions do quite well, especially the GEM.
\end{abstract}




\section{INTRODUCTION}

It is our intention here on the occasion of John Cowley's 70th birthday to review various aspects of the current siatus of short-range order in alloys especially as applied to the determination of effective pair interactions (EPI's) from diffuse $X$-ray or neutron scattering. While this enterprise began essentially with the first single crystal study of short-range order in Cu3Au by Cowley in 1950 [1] it has flourished since then and has become a principal method for studying disordered alloys. Accompanying the development of newer techniques to collect and analyze alloy diffuse scattering data, and to extract the underlying energetics of alloy phase formation, has been a concomitant development of first principles electronic structure calculations for disordered alloys (see, for example, the book by Ducastelle [2] or the proceedings of the recent NATO ASI on "Static and Dynamics of Alloy Phase Transformations" [3]).

Following Cowley's pioneering work on this subject came several papers in which the scattering effects of the size disparity between the constituent atoms of a binary alloy were developed. These effects consisted of a) an enhanced diffuse intensity about the fundamental Bragg peaks, referred to as Huang scatterıng after an early paper by K. Huang [4] on lattice displacements in dilute alloys, which arises from terms in the scattering equations which are quadratic in these displacements and b) an asymmetric contribution to the diffuse scattering, often referred to as the "size effect" [5]. There now exists a number of treatments for separating these contributions including, most notably, the widely used method of Borie and Sparks [6] and more recently the papers of Dietrich and Fenzl [7] 
who use a cumulant expansion scheme to reveal the diffraction effects in a rather complete way.

There is also an extensive parallel treatment of alloy diffuse scattering by Krivoglaz [8] who develops all of the relevant scattering formalism directly in k-space (as opposed to the r-space treatments noted above) in which the contribution of concentration fluctuations (short-range order) and the attendant displacement waves are explored.

In the present report we shall confine ourselves to the (symmetric) short-range order scattering originally measured by Cowley [1]. The accompanying atomic displacements are fascinating in themselves and can, through the Fourier transform of the elastic energy contributed by them, be directly incorporated as a strain contribution to the EPI's $[8,9]$. In fact, as we discuss the EPI's, we will note that these interactions often persist to distant neighbors in an alloy (more, say, than the first two coordination shells) and that this tendency is due both to long-range electronic and elastic effects and to electronic screening of the charge or valence fluctuations in an alloy (Friedel oscillations). However, in our simulations for a binary bcc alloy in Section 3 below, we will confine ourselves to the nearest neighbor interaction, $V_{1}$, both for simplicity's sake and because the physics of interest is clearly revealed.

The EPI's in question are usually written as:

$$
\left.V_{m n}=1 / 2 V_{m n}^{A A}+V_{m n}^{B B}-2 V_{m n}^{A B}\right]
$$


where $\vec{P}_{m n}$ connects the atoms on two lattice sites $(m, n)$ in the crystal and $V_{m n}$ is their effective pair interaction, analogous to the exchange interaction of magnetism. (We might also construct triplet and higher order interactions, and these are indeed used, but we shall here retain the convenient simplification of pairs. The concentration dependence of $V_{\mathrm{mn}}$ may be shown to yield many of the consequences of the higher order terms.) The Warren-Cowley (or Cowley-Warren) short-range order parameters, or pair correlation functions, introduced by Cowley [1] are written as:

$$
\alpha_{m n}=1-\frac{P_{m n}^{A / B}}{C_{A}}, \quad\left[c_{A}=c, c_{B}=(1-C)\right]
$$

where $P_{m n}^{A B B}$ is the conditional probability that an $A$ atom is on site $m$ given a $B$ on site $n$, with the concentration $\mathrm{CA}_{\mathrm{A}}=\mathrm{c}$ being the random value. For $\mathrm{a}$ random alloy $\alpha_{m n}=0$ for all $\vec{r}_{m n}$, except $m=n(\vec{p}=0)$ for which $\alpha_{\infty}=1.0$ (an $A$ atom cannot sit on a B origin site). The X-ray scattering per atom, neglecting all of the atom size contributions - especially as they can now effectively be separated either experimentally or theoretically - is given by

$$
I(\vec{k})=\frac{1}{N} c(1-c)\left(f_{A}-f_{B}\right)^{2} \sum_{m n} \alpha_{m n} e^{i \vec{k} \cdot \vec{r}_{m n}}
$$

where $f_{A, B}$ are $X$-ray or neutron scattering factors and $N$ is the number of atoms. Normalizing Eq.(1) we have

$$
\frac{I(\vec{k})}{c(1-c)\left(f_{B}-f_{A}\right)^{2}}=\alpha(\vec{k})=\frac{1}{N} \sum_{m n} \alpha_{m n} e^{i \vec{k} \cdot \vec{r}_{m n}}
$$


which for primitive disordered alloys is clearly a symmetric scattering function which repeats in each Brillouin zone of reciprocal space.

Similarly we can write

$$
V(\vec{k})=\frac{1}{N} \sum_{m n} V_{m n} e^{i \vec{k} \cdot \vec{r}_{m n}}
$$

for the Fourier transform of the EPI's. The meaning of $\mathrm{V}_{\mathrm{oo}}\left(\equiv \mathrm{V}_{0}\right)$ is somewhat less trivial than $\alpha_{00}=1$ and in fact is related to the self energy of the alloy and the heat of mixing as we shall see. Again, as with the $\alpha_{m n}$ for a simple disordered alloy, $V_{m n}=V_{1}$ if $\vec{P}_{m n}$ connects (equivalent) nearest neighbors, $V_{m n}=V_{2}$ for second neighbors and so forth. We shall use $\alpha_{s}$ and $V_{s}(s=m-n)$ interchangeably with $\alpha_{m n}$ or $V_{m n}$ wherever clarity is not sacrificed.

In Section 2 which follows we compare various approximate expressions, including those originally derived by Cowley [10] and rederived by Clapp and Moss [11], in which the $\alpha_{m n}$ are related to the $V_{m n}$ $[(\alpha, \vec{k})$ is related to $V(\vec{k})]$. All of these expressions fall within the general class of mean-field theories for the fluctuations which, as Brout [12] has noted, become increasingly accurate above $1 / z$ of the critical temperature for ordering, where $z=$ nearest neighbor coordination number. These theories are mean-field in the way that they replace the true fluctuating field that any atom sees with an averaged or mean field. In essence, the direct correlation between an origin site $o$ and its nth neighbor, $\alpha_{o n}$, is replaced by 
the correlations between site $o$ and the $f$ neighbors of $n$ and the pair interaction of these $f$ sites with $n, V_{f n}$. The fluctuations in these interactions are then replaced by the mean value. This approximation can, however, be quite effectively "patched up", as we shall see.

There also exists an "exact" scheme for an arbitrary range of interaction developed by Gerold and Kern [13] in which an Inverse Monte Carlo Method (IMC) is used with the measured values of $\alpha_{s}$ to calculate $V_{s}$. This has been employed over the past few years with great success and it is our final task in Section 4 to compare the IMC values of $V_{S}$ from recent experimental scattering results on $\alpha_{s}$ with several of the approximate schemes. It will be seen that these approximate schemes in general do rather well when compared with the IMC.

\section{THEORETICAL DEVELOPMENT}

In extracting the pair interactions from experimental diffuse scattering data the Krivoglaz-Clapp-Moss (KCM) $[8,11]$ approximation is widely used because of its simplicity:

$$
\alpha(\vec{k})=\frac{D}{1+2 \alpha(1-c) \beta V(\vec{k})}
$$

Here, $V(\vec{k})$ and $\alpha(\vec{k})$ are, as noted earlier in Eqs.(2) and (3), the Fourier transforms of the SRO parameters $\alpha m n$ and the pair interactions $V_{m n}$, respectively, $c$ is the concentration, $\beta=(k B T)^{-1}$ is the inverse temperature, and $D$ is a normalization constant. As can be easily seen from 
Eq. (4), the value $D=1$ used in Krivoglaz' derivation [8] violates the sum rule $\alpha_{0}=1$ when applied to the "direct problem" of determining the SRO parameters from the interactions and yields a non-zero self-interaction $V_{0}$ when used to solve the "inverse problem," with which we will be concerned here. Several other choices have been proposed for D. Clapp and Moss [11] used

$$
D=\Omega^{-1}\left[\int d \vec{k} \frac{1}{1+2 c(1-c) \beta V(\vec{k})}\right]^{-1}
$$

which satisfies $\alpha_{0}=1$, whereas Lefebvre et al.[14] introduced

$$
D=\left[\Omega^{-1} \int d \vec{k} \alpha^{-1}(\vec{k})\right]^{-1}
$$

which yields $V_{0}=0$.

Recently, Masanskii et al. [15] developed a theory of SRO in alloys based on the $\gamma$-expansion method (GEM)[16]. Their formalism treats the Ising model by means of an expansion in powers of $\gamma=\exp (-1 / \xi)$, where $\xi$ is the correlation length. The underlying assumption is that the pair correlation function $G_{m n}=c(1-c) \alpha_{m n}$ falls off exponentially with the interatomic distance $r m n$ for systems not too close to critical points. The expansion is applied to the general relation linking correlations with interactions, $G=(\beta \vee-\Sigma)^{-1}$ ( $\Sigma$ is the self-energy operator). For cubic lattices one then obtains an explicit solution to the inverse problem in terms of the 
approximate KCM result [Eq. (4)] and the off-diagonal part of the selfinteraction $\Sigma[15,17]$ :

$$
V_{S}=\frac{1}{D} V_{S}^{K C M}+\frac{1}{2} k_{B} T \Sigma_{S}, s \neq 0
$$

with

$$
\begin{aligned}
& \Sigma_{1}=A \alpha_{1}^{2}+B \alpha_{1}^{3} \\
& \Sigma_{2,3}=A \alpha_{2,3}^{2} \\
& \Sigma_{s}=0 \quad s>3,
\end{aligned}
$$

where

$$
\begin{aligned}
& A=\frac{1}{2} \frac{(1-2 c)^{2}}{[d(1-c)]^{2}} \\
& B=\frac{1}{6} \frac{[1-6 d(1-c)]^{2}-3(1-2 c)^{4}}{[d(1-c)]^{3}}
\end{aligned}
$$

The GEM theory was shown to be very ascurate in comparison with the exact Inverse Monte Carlo Method (IMC) [13] (see Ref. [15] and the discussion below). One consequence of Eq. (7) is that the Krivoglaz value $D=1$ in the $\mathrm{KCM}$ equation provides the best agreement with the exact result, whereas the choices in Eqs. (5) and (6) tend to underestimate the magnitude of the interactions [15]. The GEM treatment also yieids an expression for $V_{0}$. To lowest order (neglecting the non-diagonal part of $\Sigma$ ) one has [17]: 


$$
V_{0}=-\sum_{s \neq 0} \alpha_{s} V_{s}
$$

This result has already been obtained by Hoffman [18] in the framework of the spherical model. Using a non-zero $V_{0}$ according to Eq. (10) and setting $D=1$ in the KCM formula indeed satisfies the normalization condition $\alpha_{0}=1$ and yields much more accurate results for the "direct problem" than the use of $V_{0}=0$ and the Clapp-Moss; normalization as in Eq.(2) [18]. Of course, when solving the "inverse problem", $V_{0}$ is of no concern and only the choice of $D$ is essential; i.e., the spherical model and the $\mathrm{KCM}$ equation with $D=1$ are identical when used to calculate $V_{S}, S \neq 0$ from the SRO parameters. Note that the GEM theory automatically satisfies $\alpha_{0}=1$ in all orders of the expansion while yielding the spherical model in the zeroth-order approximation, $\Sigma_{S}=0$ for $\$ \neq 0$ [17].

The Cowley theory of ordering in alloys [10] was first derived assuming approximate expressions for both the configurational energy and entropy and, as noted earlier, was rederived in a more general framework by Clapp and Moss [11].

The Cowley equations, with the index "o" referring to an origin site, are

$$
2 \sum_{m} V_{n m} \alpha_{o m}+k_{B} T f\left(\alpha_{o n}\right)=0
$$

with 


$$
\begin{aligned}
& f\left(\alpha_{o n}\right)=\ln \left\{\frac{\left(\frac{c}{1-c}+\alpha_{\text {on }}\right)\left(\frac{1-c}{c}+\alpha_{\text {on }}\right)}{\left(1-\alpha_{o n}\right)^{2}}\right\} n \neq 0 \\
& f\left(\alpha_{o n}\right)=\frac{\alpha_{\text {on }}-D}{d(1-c)}, \quad n=0
\end{aligned}
$$

Here, the term for $n=c$ (not present in Cowley's original treatment [10]) has been included to ensure compatibility with the KCM formula, Eq. (4) . Expanding $f\left(\alpha_{o n}\right)$ to lowest (linear) order in $\alpha_{o n}, n \neq 0$, one obtains (for all $n$ )

$$
2 \sum_{m} V_{m m o m}+\frac{k_{B} T}{d 1-c)} \alpha_{o n}=\frac{D \delta_{o n} k_{B} T}{c(1-c)}
$$

which in fact is the KCM equation. Note that the interactions enter Eqs.(11) only linearly in the convolution product thus allowing a straightforward diagonalization via Fourier transformation when solving the "inverse problem". The direct problem, i.e. obtaining $\alpha_{\mathbf{s}}$ from $V_{\mathbf{S}}$, however, requires a solution of a coupled set of non-linear equations.

An expansion of the logarithm in Eq.(11b) up to quadratic terms in $\alpha o n$, followed by a Fourier transformation and expansion in powers of $\alpha(\vec{k})-1$ (assuming $\alpha_{0}=1$ ) gives the lowest-order result for the difference between the KCM and the Cowley predictions:

$$
V_{s}^{\text {Cowley }}=V_{s}^{K C M}+\frac{1}{2} k_{B} T A \alpha_{S}^{2} \quad(s \neq 0)
$$


with $A$ from Eq. (9a). Therefore, for $D=1$ the Cowley expression gives the same lowest-order correction to the KCM interactions as the GEM theory. (At least for the first 3 shells; the validity of the approximations used here to derive Eq. (13) becomes more uncertain for higher shells, s.)

Going beyond the quadratic approximation in Eq. (11) results in more intricate expressions which, in general, cannot be solved in a closed form. However, in the limiting case $\alpha_{s} / \alpha_{1} \rightarrow 0, s>1$, i.e., for a vanishing correlation length $\xi \rightarrow 0$, one easily obtains an asymptotically correct expansion for the nearest-neighbor interaction $V_{1}$ :

$$
V_{1}^{\text {Cowley }}=V_{1}^{K C M}+\frac{1}{2} k_{B} T\left(A \alpha_{1}^{2}+B^{\prime} \alpha_{1}^{3}\right)
$$

where $B^{\prime}$ is given by

$$
B^{\prime}=\frac{-9[d(1-c)]^{2}+6 d(1-c)-1}{[d(1-c)]^{3}}
$$

In Fig. 1 the quantities $A[c(1-c)]^{2}, B[c(1-c)]^{3}$ and $B^{\prime}[c(1-c)]^{3}$ are plotted as function of the concentration. We show these combined quantities rather than $A, B, B^{\prime}$ because the SRO parameters entering Eqs. (8) and (14) are asymptotically proportional to $\mathrm{c}(1-\mathrm{C})$, as in the KCM formula for $c(1-c) \beta \rightarrow 0$. Figure 1 shows that whereas $B[c(1-c)]^{3}$ and $\mathrm{B}^{\prime}[\mathrm{C}(1-\mathrm{c})]^{3}$ are identical in the limit $\mathrm{c} \rightarrow 0$ they differ considerably at higher concentrations. In particular $B$ and $B^{\prime}$ have different signs at $c=0.5$ where $A$, and therefore the lowest order correction, quadratic in $\alpha_{1}$, vanishes. Since the GEM theory has proven to be very accurate, this indicates that the 
Cowley expression should be a good approximation at low concentrations but could deviate from the exact result even more than the KCM theory around the equicomposition, $c=0.5$. We will substantiate this suggestion in the next section.

\section{MONTE CARLO SIMULATIONS}

In order to compare the validity of the different approximations to the exact "inverse" Ising problem presented above we have performed a series of Monte Carlo calculations with an assumed Ising Hamiltonian on the bcc lattice. For the sake of simplicity we have restricted the Hamiltonian to contain only a positive nearest-neighbor pair interaction, $V_{1}$. The resulting phase diagram exhibits a A2 to $B 2$ second order phase transition with a critical temperature $T_{c}=3.245 V_{1}$ at the equiatomic composition [19]. The Monte Carlo simulations were done using the Kawasaki spin-exchange algorithm, i.e., in the canonical ensemble on a bcc lattice containing $32 \times 32 \times 32$ atoms. The size of the model was found to be sufficiently large compared with the correlation lengths for all temperatures and concentrations studied thus minimizing finite-size effects. To improve efficiency, the three-dimensional crystal was mapped onto a linear chain with helical boundary conditions [20]. Starting from the totally random distribution, 2000 initial Monte Carlo steps (MCS) per atom were discarded in order to avoid the influence of the initial conditions. The thermal averaging was performed during subsequent 1000 MCS. Several long-time runs up to 12000 MCS were used to verify that equilibrium was indeed obtained. At every point in the $(\mathrm{c}, \mathrm{T})$ field the statistical averages for the 
first 20 pair correlation functions were calculated (up to the 551 shell). The values thus obtained were used to calculate the pair interactions with the methods discussed in Section 2. Following this discussion, the value $D=1$ was employed in all evaluations. Figure 2 shows the results as function of the reduced temperature $T / T_{c}$ where $T_{c}$ is always the equiatomic $(c=0.5)$ critical temperature. The recalculated pair interactions have been normalized to the input value of $V_{1}$ used in the Monte Carlo simulations, i.e., the exact solution of the inverse problem is $V_{1}=1$ and $V_{S}=0$ for $s>1$.

At the equiatomic composition (see Fig. 2a) both the KCM and Cowley theories overestimate $\mathrm{V}_{1}$. However, the $\mathrm{KCM}$ approximation is more accurate since the Cowley value for $V_{1}$ is systematically higher than the $\mathrm{KCM}$ one. The simulation thus confirms the prediction of our approximate power-series expansion at $\mathrm{c}=0.5$, where the second-order corrections to the KCM theory vanish: the Cowley third order $\left(\sim \alpha_{1}^{3}\right)$ "correction" for $c=0.5$ actually worsens the result if one takes the GEM expressions as a measure of accuracy. The GEM corrections to the KCM values are indeed very reliable, except in the vicinity of the critical point.

The KCM approximation becomes increasingly less reliable at lower concentrations where it underestimates $V_{1}$ (see Figs. $2 b, c$ ). As pointed out in [15] this underestimation can be explained by the invariably positive second order GEM correction, see Eqs. (7) and (8), if this correction dominates other terms (this will be most likely the case for small concentrations). The Cowley results still overestimate $V_{1}$, but give a better overall agreement. Note, however, that the Cowley equations yield increasingly positive values for (non-existent) more distant interactions at 
low $c$ and $T$ (see $V_{2}$ in Figs $2 b-c$ ). The magnitude of these interactions is smaller when using the KCM approach. The accuracy of the GEM theory is again confirmed.

The results of the KCM and the Cowley theory are summarized in Fig. 3 which shows the concentration dependence of $\mathrm{V}_{1} \mathrm{KCM}$ and $\mathrm{V}_{1}$ cowley for different temperatures. The worsening of the $\mathrm{KCM}$ approximation and the improvement of the Cowley result with decreasing concentration are clearly visible. For every temperature (above the critical line) $V_{1}$ in the Cowley theory seems to converge towards the exact result in the dilute limit $c \rightarrow 0$. The deficiency of the KCM equation for $\mathrm{c} \rightarrow 0$ is somewhat surprising at first glance because it is tempting to regard $\beta c(1-c)$ as the "small parameter" of the KCM theory. However, Eqs. (7-9) already suggest that the KCM approximation does not yield the exact result for $c \rightarrow 0$ and constant $\beta$.

Since $V_{1}{ }_{1} \mathrm{KCM}>1$ for $\mathrm{c}=0.5$ and $\mathrm{V}_{1} \mathrm{KCM}<1$ for $\mathrm{c} \rightarrow 0$ there must be a $\operatorname{cex}(T)$ for which the $K C M$ theory recovers the exact result, $V_{1}=1$. Furthermore, from the form of the GEM equations one expects that $c_{e x}$ will be only weakly dependent on the temperature. From Fig.1, cex must lie between $\mathrm{c}=0.5$ (where the second-order GEM correction vanishes) and $\mathrm{c} \approx 0.3$ (where the third-order GEM correction changes sign). In our simulation cex seems to be close to 0.4 (see Fig. 3). This would imply that for determining $V_{1}$ in ordering systems, the $\mathrm{KCM}$ approximation is at its best around $40 \%$ solute concentration. Note that this "crossoverphenomenon" does not occur in phase-separating alloys because for $\alpha_{1}>0$ the KCM theory underestimates $V_{1}$ both at $c=0.5$ and for $\mathrm{c} \rightarrow 0$, as can be inferred from Eqs. (7-9). Similarly, a comparison of Eqs. (9b) and (15) - 
again under the assumption that the GEM result is a good approximation to the exact solution - shows that the Cowley theory underestimates $V_{1}$ for all $C$ in systems with a tendency toward phase segregation $\left(\alpha_{1}>0\right)$ and overestimates $V_{1}$ in ordering systems $\left(\alpha_{1}<0\right)$.

\section{COMPARISON WITH EXPERIMENT}

We present here a selection of recent $X$-ray and neutron diffuse scattering data on short-range order in binary alloys. These data sets have either been corrected for atom size-effects, including the quadratic displacement terms and the linear cross terms referred to earlier as the "size-effect", or have been corrected so as to eliminate at least the linear terms. From these data sets the pure symmetric short-range order intensity has then been determined and its Fourier inversion performed to obtain directly an extensive set of parameters, $\alpha_{s}$. These sets of $\alpha_{s}$ were then used to recreate characteristic ensembles of disordered alloy, as first done by Gehlen and Cohen [21]. The IMC was then applied to several of these re-constructed alloys to determine an averaged set of the first tive values of $V_{s}(s=1,5)$. These $I M C$ results are what we refer to as "exact". The KCM, Cowley and GEM solutions for $V_{s}$ were also done as described earlier in

Section 2: $\alpha(\vec{k})$ was solved for $V(\vec{k})$ which was then Fourier transformed to get $V_{s}$. This is easily done for the KCM and Cowley expressions, and the GEM corrections to KCM may be simply performed, as noted earlier; in all cases, $D=1$.

The first alloy system in Fig.4(a) is Fe0.53Cr0.47 recently studied by Reinhard et al. [22]. This is a bcc alloy of the clustering variety which was investigated using anomalous scattering at the NSLS synchrotron radiation 
facility at the Brookhaven National Laboratory. A single crystal was quenched from just above the miscibility gap, below which there is an intervening $\sigma$-phase which forms only on long annealing. The diffuse scattering was measured at three energies, one of which essentially removed the contrast between $\mathrm{Fe}$ and $\mathrm{Cr}, \mathrm{IfFe}_{\mathrm{f}} \mathrm{fcrl} 0$, and permitted the removal of all quadratic scattering effects associated with the average lattice, such as Huang and thermal diffuse scattering. What remained was least-squares analyzed for short-range order and the "size-effect." Figure 4(a) shows clearly that in this case of weak short-range order (clustering, $V_{1}<0$ ) all $V_{S}$ save $V_{1}$ are small, but measurable, and the IMC value of $V_{1}$ is slightly more positive than the Cowley and KCM values. All of the GEM values are indistinguishable from the IMC points. $V_{2}$ is actually still appreciable while $V_{3}-V_{5}$ are nearly zero and $V_{4}$ and $V_{5}$ must, in any case, be equal for the KCM and GEM solutions. The values for $V_{1}$ and $V_{2}$ are also in excellent agreement with a KKR-CPA-GPM first principles calculation of Turchi and co-workers [23].

For our second comparison we present in Fig.4(b) the results of a recent neutron study by Reinhard and co-workers [24], of a disordered fcc Cu0.69Zno.31 crystal, using isotopically substituted $65 \mathrm{Cu}$ to enhance the contrast. In this case a triple axis spectrometer, operating at zero energy transfer, removed the one-phonon scattering (TDS). The pure short-rangeorder scattering, extracted by a least squares fitting procedure, showed not only the expected diffuse peaking about the forbidden fcc positions, such as (100) and (110), but a pronounced enhancement of diffuse "satellites" at incommensurate positions given by $2 \mathrm{KF}_{\mathrm{F}}$ as first suggested by Krivoglaz [8] and demonstrated by Moss [25] for Cu-Au alloys. These effects certainly 
require, via Eq.(4), a long-range interaction of an oscillatory type in directions in the crystal parallel to Fermi surface flats or nesting features spanned by $2 \mathrm{kF}$ (i.e. in [110] directions). However, in the present comparison we do not concern ourselves with this aspect of the diffuse scattering and of $V_{s}$.

Figure $4(\mathrm{~b})$ shows clearly, as discussed in Section II, that the Cowley theory overestimates $V_{1}$ and $V_{2}$ while KCM slightly underestimates both. The GEM is again essentially identical to the IMC results for $V_{1}$ and $V_{2}$. For $V_{3}-V_{5}$ all treatments give the same results which, again, for KCM and GEM, is required for all $V_{s}, s>3$.

The next comparison is with recent short-range order measurements on $\mathrm{Cu}_{3} \mathrm{Au}$ by Butler and Cohen [26]. Such measurements were made originally by Cowley [1] followed by Moss [27] and Bardhan and Cohen [28], but we believe the Butler-Cohen data to be the most reliable. [The $\alpha_{s}$ values are also rcmarkably close to the original Cowley values!] The data were collected at $703 \mathrm{~K}$ or $35 \mathrm{~K}$ above the first-order phase transition point of $395^{\circ} \mathrm{C}$. $T / T_{C}$ is thus $\sim 1.05$ and would seem to violate Brout's criterion [12] for the validity of a mean-field expression. However, for a first-order transition this criterion is not really applicable because the fluctuations do not diverge at the measured $T_{c}$.

The method of analysis in this case was due to Georgopoulos and Cohen [29] in which, in the Borie-Sparks equations [6], advantage is taken of the difference in the scattering wavevector $(k)$-dependence of the $\mathrm{fCu}_{\mathrm{C}}$ and $f_{A u}$ to separate the various contributions to the diffuse intensity. A reliable 
self-consistent set of $\alpha_{s}$ was thereby obtained which we used as above to calculate $V_{s}$. The results are illuminating. Because the interactions are somewhat larger and longer ranged, the trends among the various treatments are more observable. The Cowley theory again overestimates $V_{1}$ and $V_{2}$ while KCM underestimates slightly these energies as noted in Section III. Beyond $V_{2}$ there is perhaps still a small enhancement of the Cowley $V_{3}$, but all schemes converge at $V_{4}$ and $V_{5}$. In any case, the worst error in the KCM for $V_{1}$ is $\sim 3 \%$ while the Cowley theory is $18 \%$ too high; i.e. the Cowley theory overcompensates for the actual fluctuating field.

Our last comparison is with the results of Schweika and Haubold [30] on a neutron study of the more dilute foc alloy Nio.89Cro.11 in which the inelastic (phonon) contribution was experimentally removed. The resulting pure short-range order scattering was obtained via a least-squares method and showed (symmetrical) diffuse peaks at positions 1 1/2 0 in the fcc reciprocal space, which is common to a variety of alloys (see the discussion in [11] and [30]). Figure $4(d)$ shows the extracted values of $V_{s}$ for this alloy. Schweika and Haubold were among the first to employ successfully the $I M C$ and to compare it with the KCM expression using $D=1$. They find that, aside from $V_{1}$, the agreement between the two is remarkable. Here we may note again the utility of the GEM and also the fact that while $V_{1}$ cowley $>$ $V_{1}$ IMC the Cowley theory yields a better $V_{1}$ for dilute alloys, albeit too large, than does the KCM. As before for small values of $V_{s}(s>2)$ they all work equally well.

Schweika and Haubold also point out that a plot of $V_{S}$ in the [110] direction reveals a long-range oscillatory behavior which, while it appeared 
to be due to Friedel oscillations, now would seem to be associated with "charge transfer" effects [31]. They also suggest that the values of $V_{s}$ may in general be used to test theories of the electronic structure of the disordered state. This prediction seems increasingly to be vindicated $[2,3,22,31]$.

\section{CONCLUSIONS}

The conclusion of this work may be briefly summarized. Beginning with the Cowley theory relating short-range order in alloys to EPI's, a large number of studies have been performed in which the effective pair energies have been evaluated using various mean field theories. With the advent of the exact Inverse Monte Carlo Method it seemed reasonable to revisit these theories both through simulation and experimental testing. Some of this comparison has already been done by Masanskii and co-workers [15] using the GEM. What we have done here is partly to popularize their conclusions. We also show via simulation how the various theories behave in various regimes of temperature and composition. Because $c(1-c) \beta$ appears combined as the "small parameter" in the KCM approximation, it would seem at first glance that this quantity, when small, would validate the mean-field treatments. That this is not true may noted directly in Figs. 2 and 3 in which the approximations fail badly at low $c$ and do rather well near $c=0.5$, especially at more elevated temperatures.

The Cowley theory yields a $V_{1}$ which is rather accurate at low $c$, but only at the expense of introducing incorrect values of $V_{2}$ and even $V_{3}$. The GEM treatment does exceptionally well, both in the simulation and in 
comparison with the IMC for experiments. As this theory provides a simple correction to the KCM treatment and vindicates Krivoglaz's choice of $D=$ ? for the normalizing constant it remains a very attractive alternative to the IMC for the evaluation of EPI's in disordered alloys.

\section{Acknowledgements}

We wish to thank Drs. David Smith and John Spence for the opportunity to participate in this Festschrift and to join our colleagues in wishing John Cowley a most happy birthday. Work at the Lawrence Livermore National Laboratory was performed under the auspices of the U.S. Department of Energy under contract No. W-7405-ENG-48. At the University of Houston this work was supported by the National Science Foundation on grant No. DMR-9208450.

\section{References}

[1] J. M. Cowley, J. Appl. Phys. 21 (1950) 24.

[2] F. Ducastelle, Order and Phase Stability in Alloys (North Holland, New York, 1991).

[3] Proceedings, NATO Advanced Study Institute on Statics and Dynamics of Alloy Phase Transformations, June 1992, Eds. P. E. A. Turchi and A. Gonis (in press).

[4] K. Huang, Proc. Roy. Soc. A 190 (1947) 102. 
[5] B. E. Warren, B. L. Averbach and B. W. Roberts, J. Appl. Phys. 22 (1951) 1493; see also B. E. Warren, X-ray Diffraction (Dover Puiplications, New York, 199c'i p. 241.

[6] B. Borie and C. J. Sparks, Acta Cryst. A 27 (1971) 198.

[7] S. Dietrich and W. Fenzl, Phys. Rev. B $\underline{39}$ (1989) 8873; ibid 8890.

[8] M. A. Krivoglaz, Theory of X-Ray and Thermal Neutron Scattering by Real Crystals (Plenum, New York 1969).

[9] H. E. Cook and D. de Fontaine, Acta Metall 17 (1969) 915; see also

D. de Fontaine in Solid State Phys. vol. 34, eds. H. Ehrenreich, F. Seitz and D. Turnbull (Academic Press, New York, 1979) p. 73.

[10] J. M. Cowley, Phys. Rev. 77 (1950) 669.

[11] P. C. Clapp and S. C. Moss, Phys. Rev. 142 (1966) 418; ibid 171 (1968) 754; S. C. Moss and P. C. Clapp, Phys. Rev. 171 (1968) 764.

[12] R. H. Brout, Phase Transitions (W. A. Benjamin, New York, 1965) p. 18.

[13] V. Gerold and J. Kern, Acta Metall. 35 (1987) 393.

[14] S. Lefebvre, F. Bley, M. Fayard, and M. Roth, Acta Metall. 29 (1981) 747.

[15] I. V. Masanskii, V.I. Tokar, and T. A. Grishchenko, Phys Rev. B 44 (1991) 4647.

[16] V. I. Tokar, Phys. Lett. 110A (1985) 453.

[17] V. I. Tokar, I. V. Masanskii, and T. A. Grishchenko, J. Phys. Condens. Matter 2 (1990) 10199.

[18] D. W. Hoffman, Metall. Trans. $\underline{3}$ (1972) 3231.

[19] N. S. Golosov and A. M. Tolstik, J. Phys. Chem. Solids $\underline{36}$ (1975) 903. 
[20] R. B. Pondey, B. Stauffer, A. Margolina, and J. G. Zabolitzki, J. Stat. Phys. 34 (1984) 427.

[21] P. C. Gehlen and J. B. Cohen, Phys. Rev. 139 (1965) A844.

[22] L. Reinhard, J. L. Robertson, S. C. Moss, G. E. Ice, P. Zschack and C. J. Sparks, Phys. Rev. B $\underline{45}$ (1992) 2662.

[23] P. E. A. Turchi, M. Sluiter and G. M. Stocks, Mater. Res. Soc. Symp. Proc. 213 (1991) 75.

[24] L. Reinhard, B. Schönfeld, G. Kostorz and W. Bührer, Phys. Rev. B 44 (1990) 1727.

[25] S. C. Moss, Phys. Rev. Lett. 22 (1969) 1108.

[26] B. D. Butler and J. B. Cohen, J. Appl. Phys. 65 (1989) 2214.

[27] S. C. Moss, J. Appl. Phys. 35 (1964) 3547.

[28] P. Bardhan and J. B. Cohen, Acta Cryst. A 32 (1976) 597.

[29] P. Georgopoulos and J. B. Cohen, J. Phys. (Paris) Colloq. 33 (1977) C7.

[30] W. Schweika and H.-G. Haubold, Phys. Rev. B $\underline{37}$ (1988) 9240.

[31] D. D. Johnson, J. B. Staunton and F. J. Pinski, Phys. Rev. B (to be published). 
Figure captions

Fig 1. Expansion coefficients in the GEM and Cowley theories (see text). solid curve : $A[c(1-c)]^{2}$, dashed curve: $B[C(1-c)]^{3}$, dotted curve: $B^{\prime}[c(1-c)]^{3}$.

Fig 2. Results for the nearest and next-nearest neighbor interactions $V_{1}$, $V_{2}$ on the bcc lattice as obtained by the various approximations in units of the $V_{1}$ used in the Monte-Carlo simulations: (a) $c=0.5$, (b) $c=0.3,(c) c=0.1$.

Fig.3 Cowley and KCM results for $V_{1}$ at different temperatures as function of concentration.

Fig. 4 Pair interactions as obtained with various methods from experimental data: (a) Fe0.53Cro.47 [22], (b) Cu0.69Zno.31 [24], (c) Cu3Au [26], (d) NiO.89Cr0.11[30]. The IMC and KCM resulis shown in (a), (b) and (d) are reproduced from [22], [24], [30]. The GEM interactions in (b) and (d) are taken from Ref. [15]. Note that in all cases $V_{S} G E M=V_{S} K C M$ for $s>3$; in all plots $a$ is the lattice parameter and $r_{s}$ is the interatomic distance. 


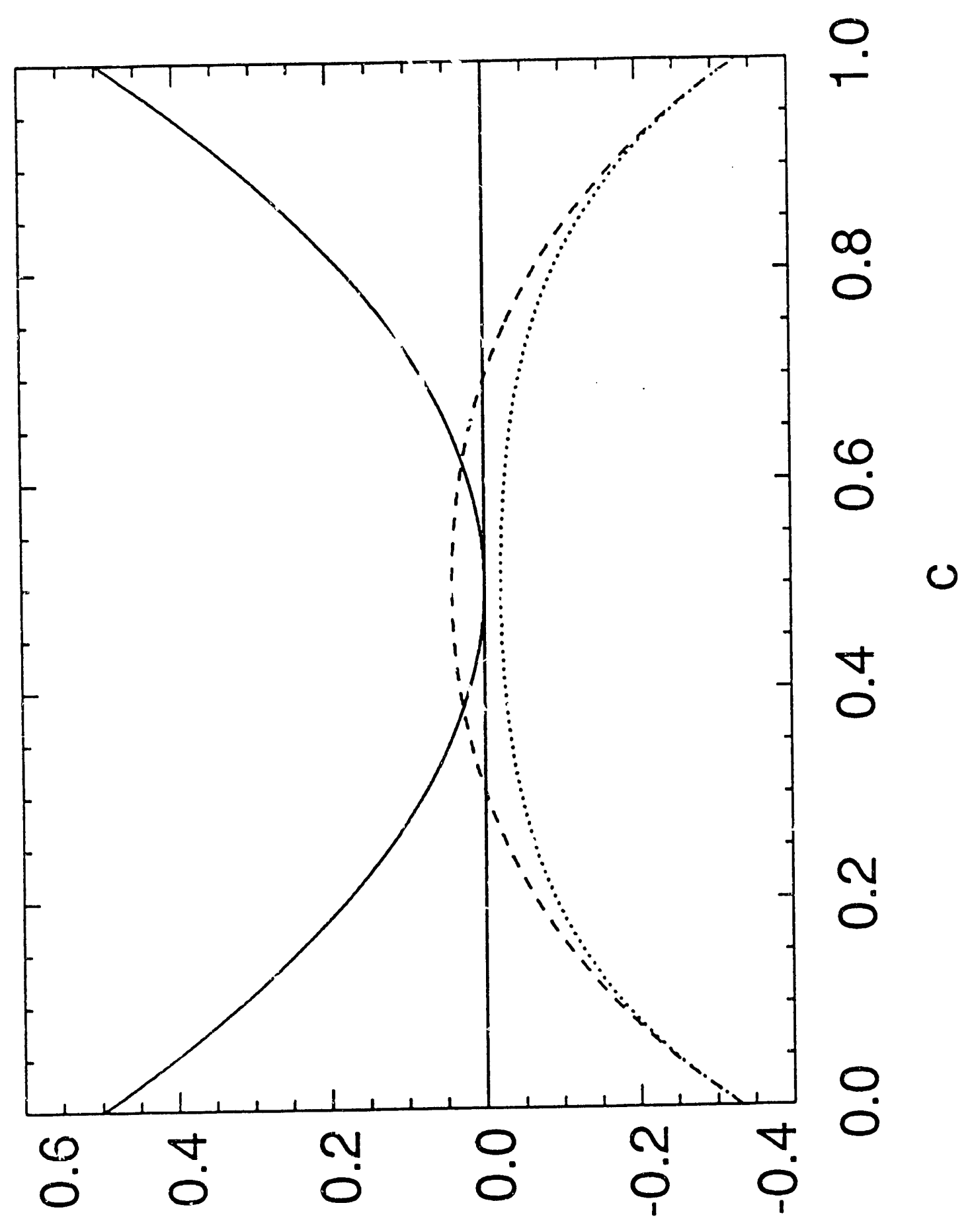




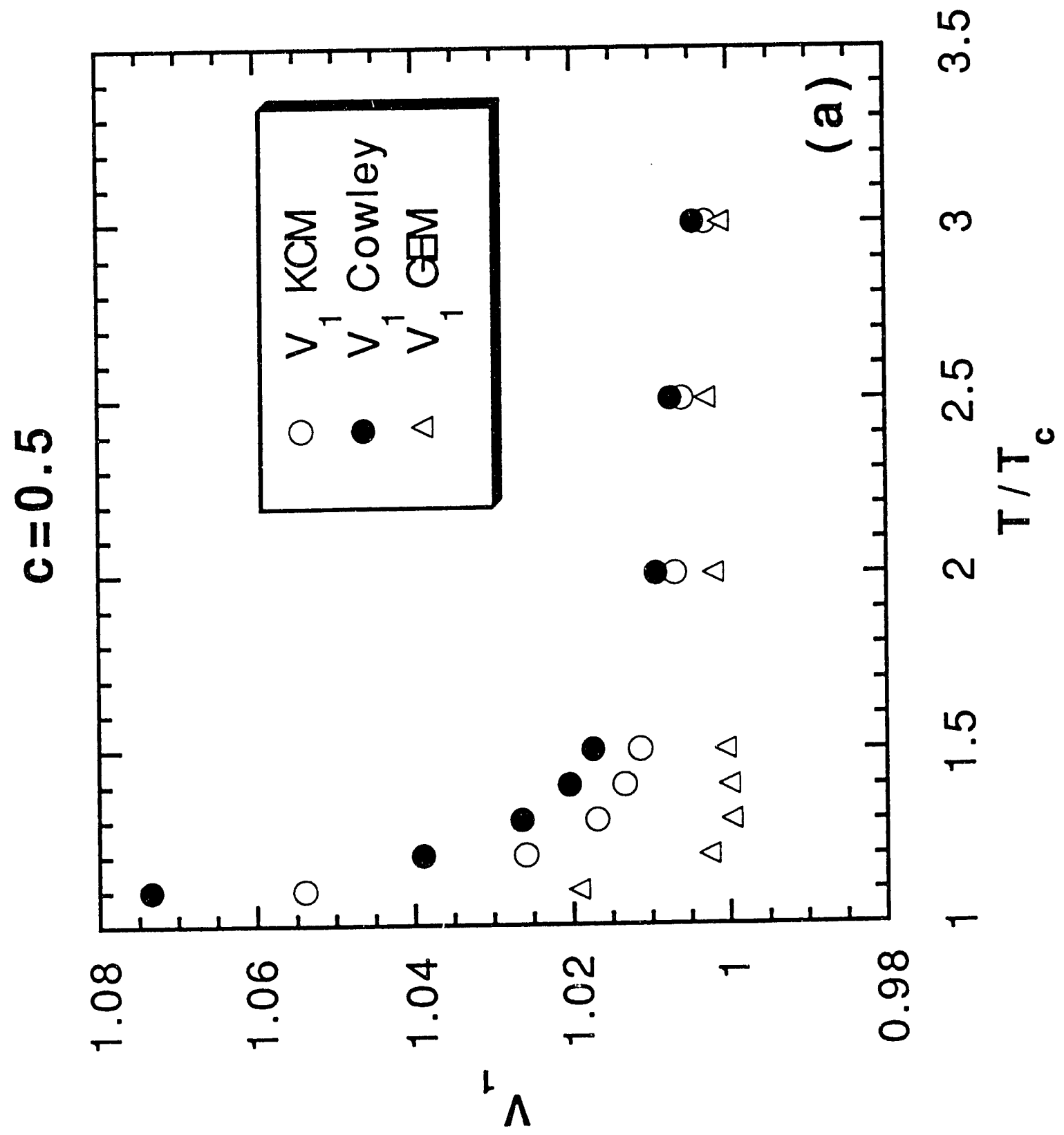




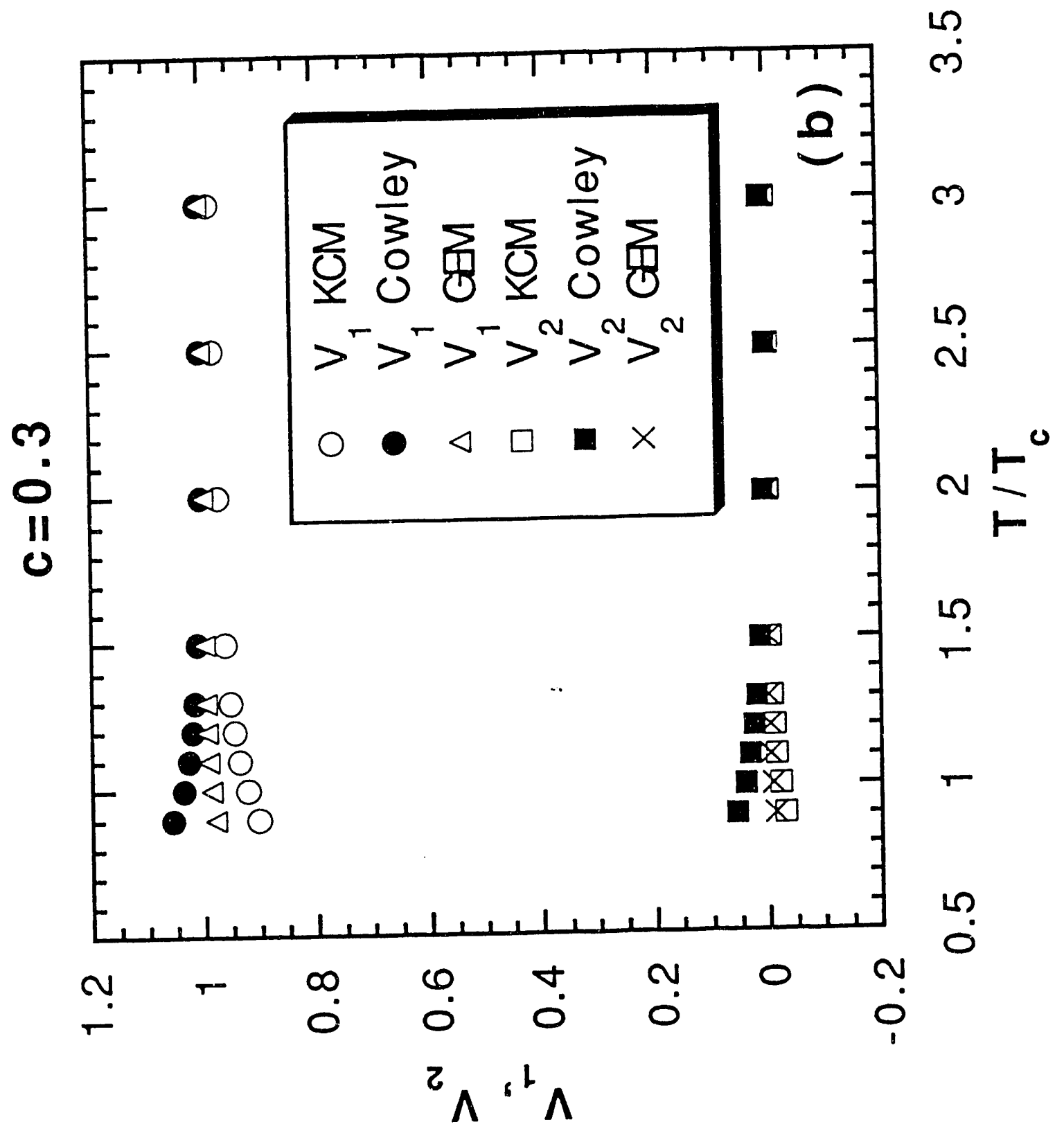




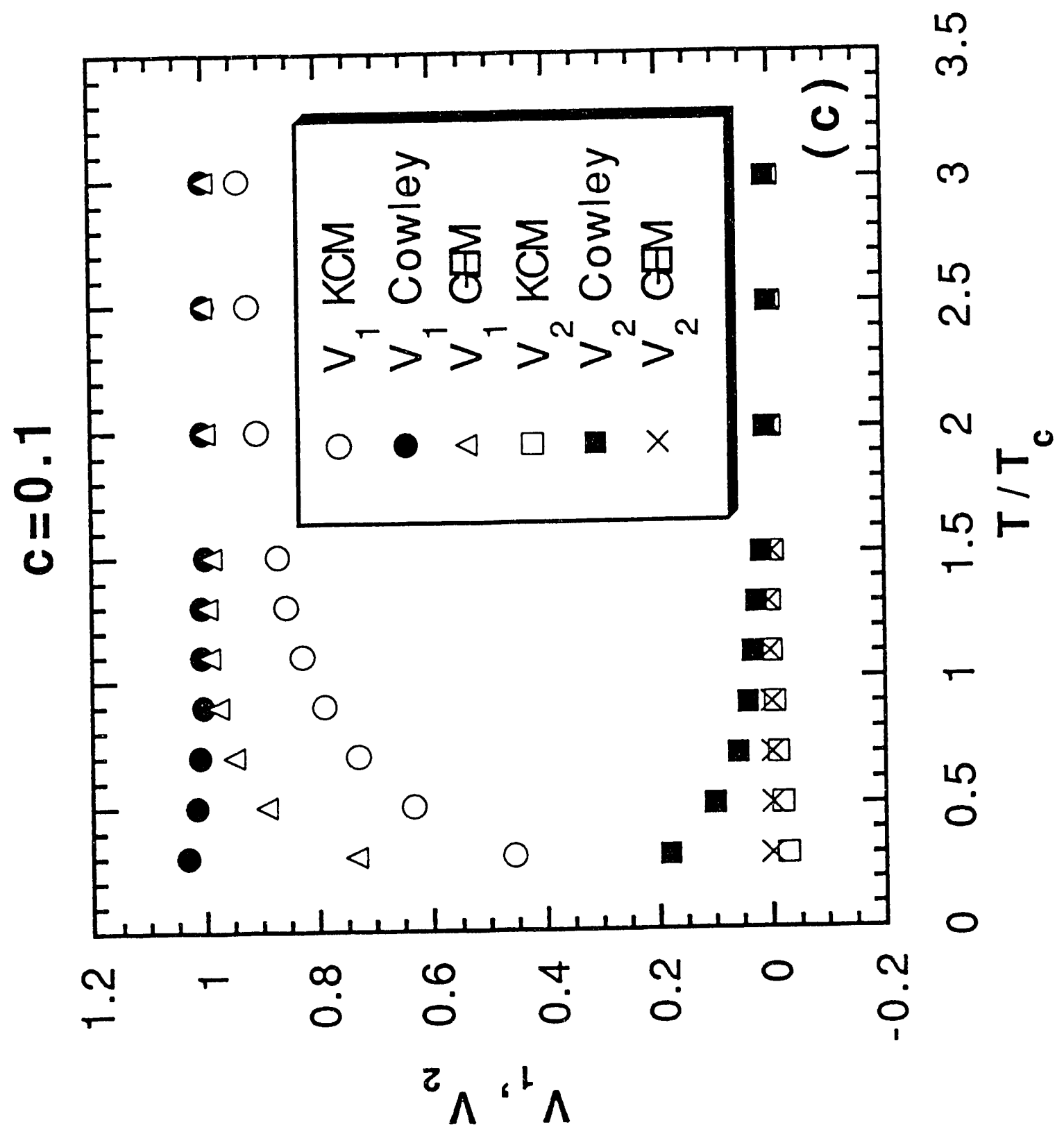




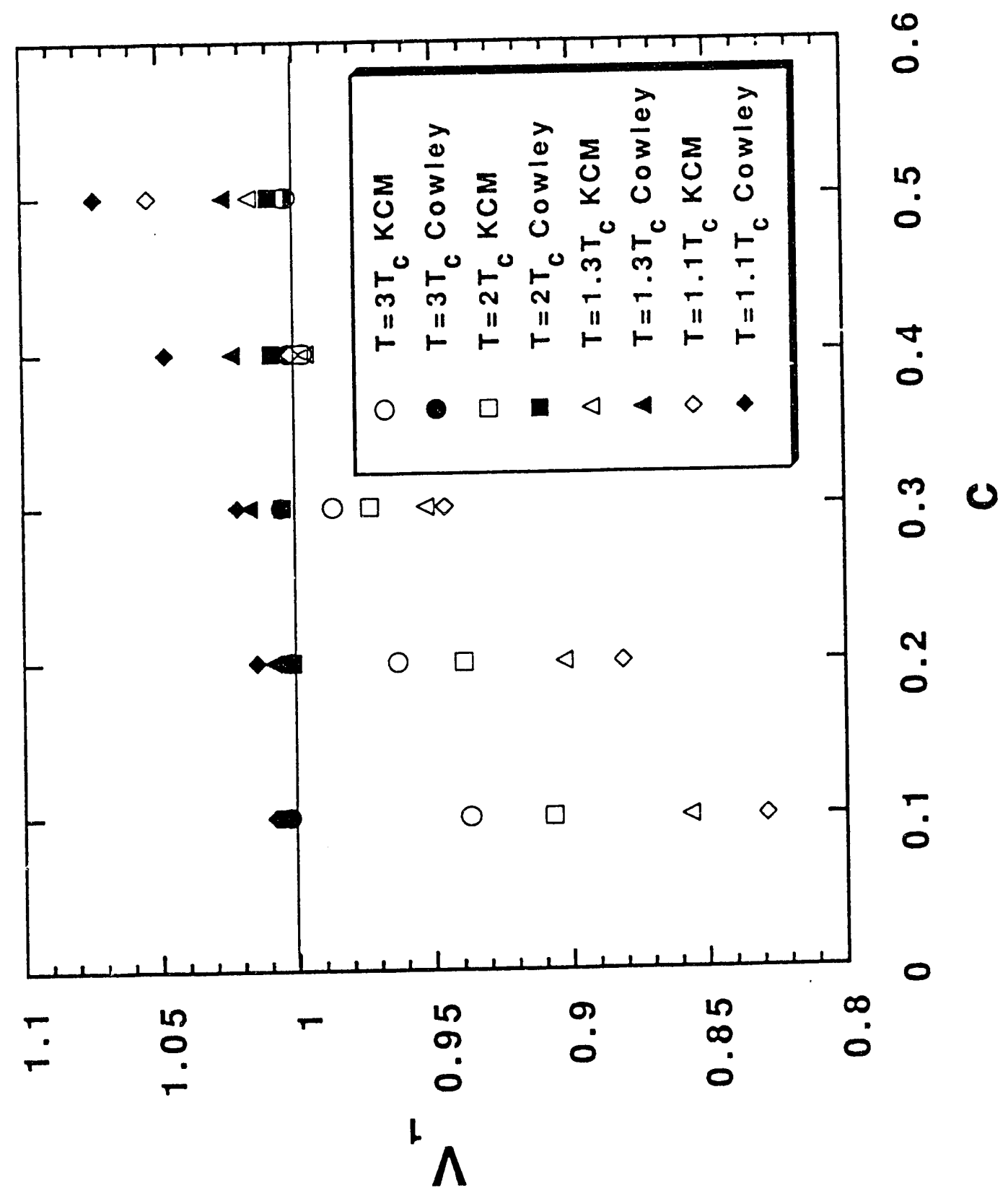




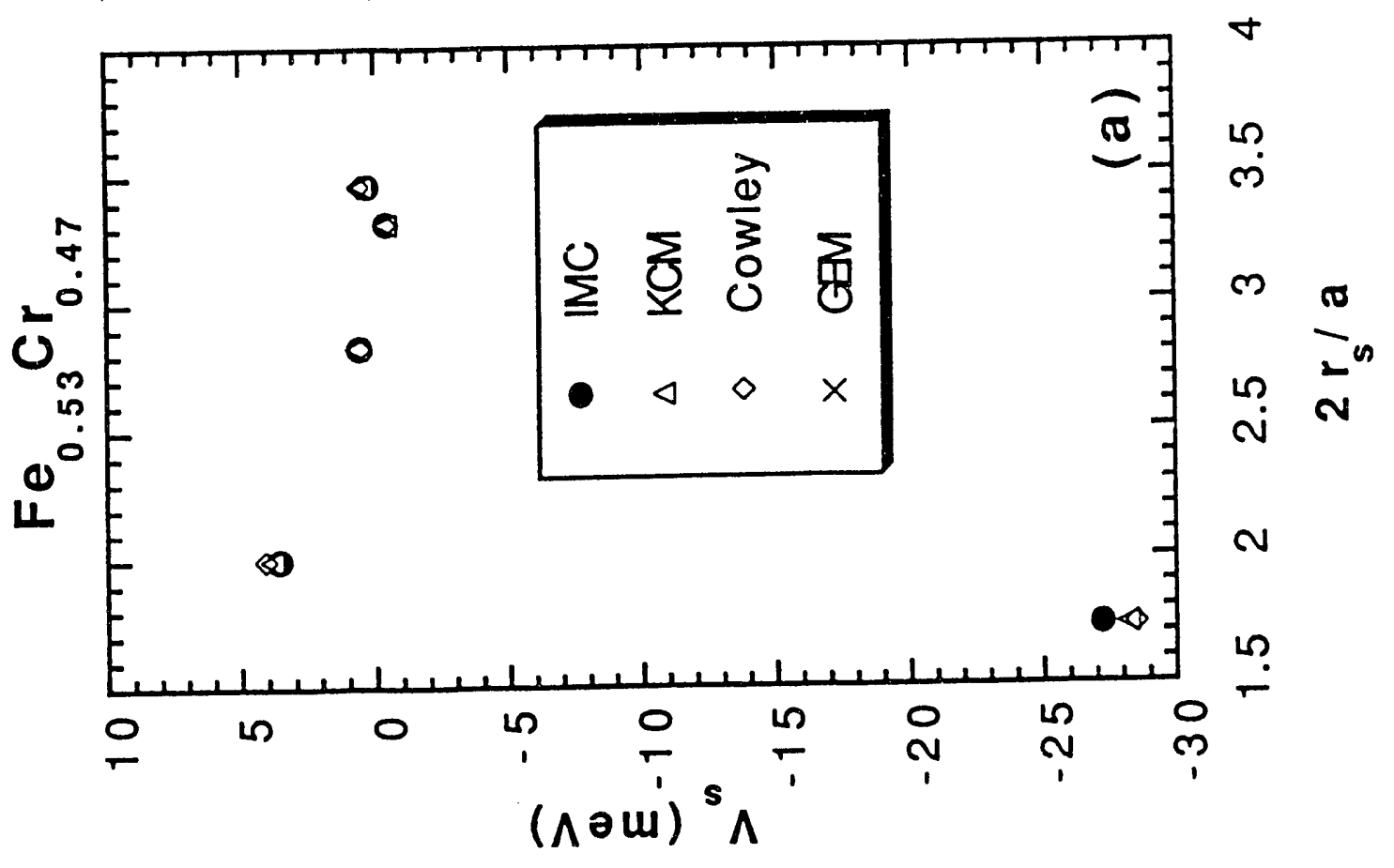




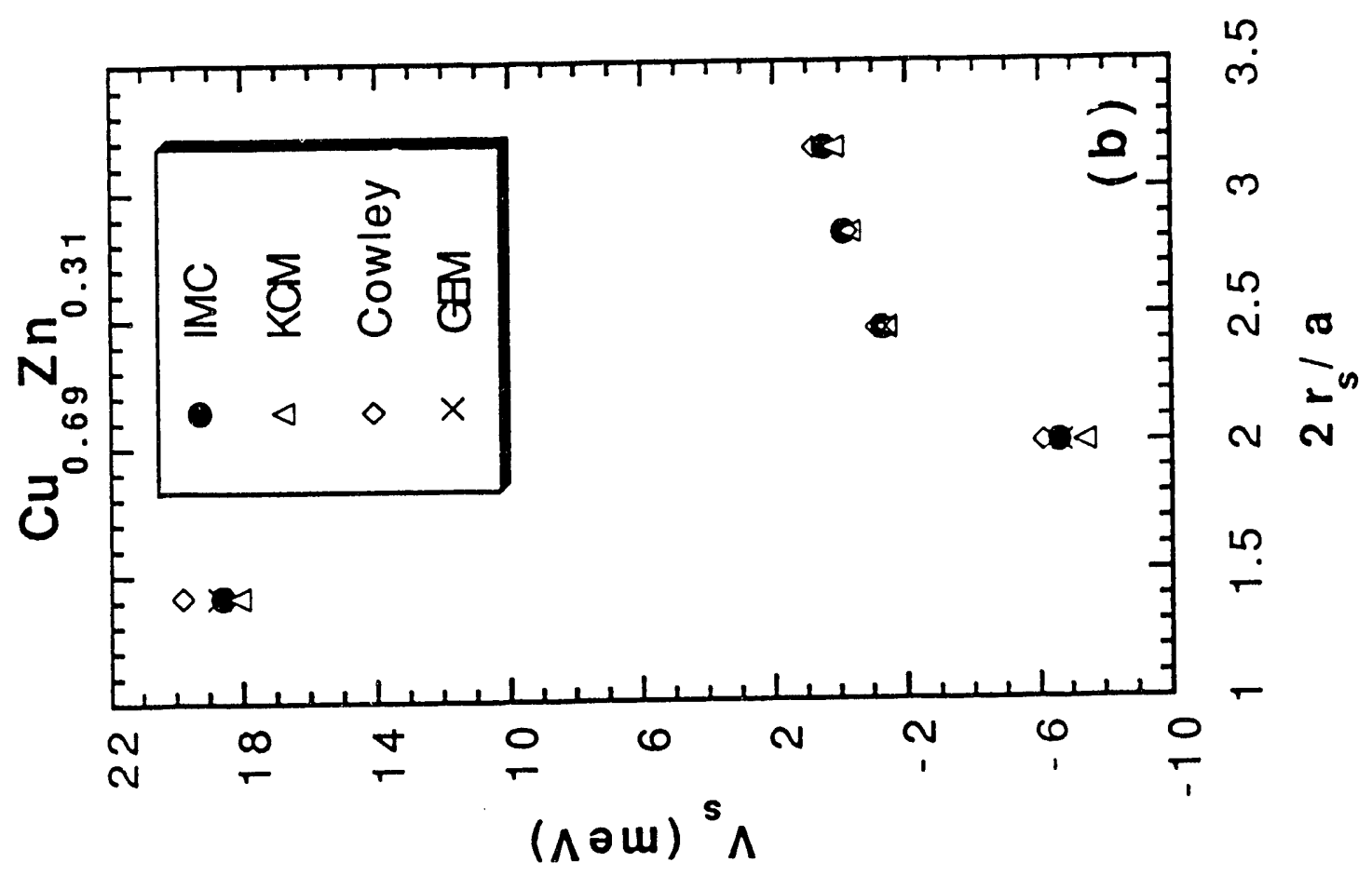




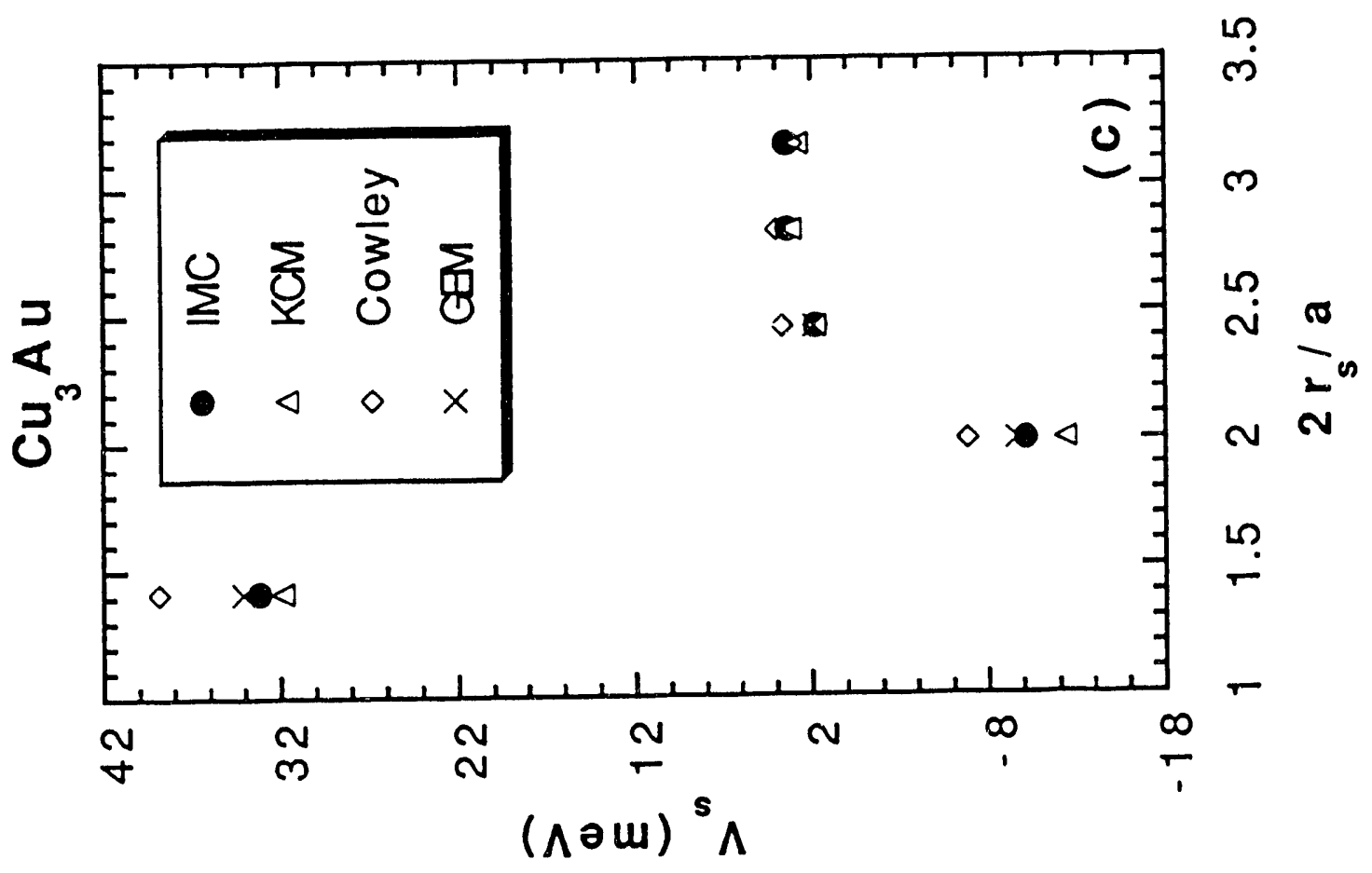




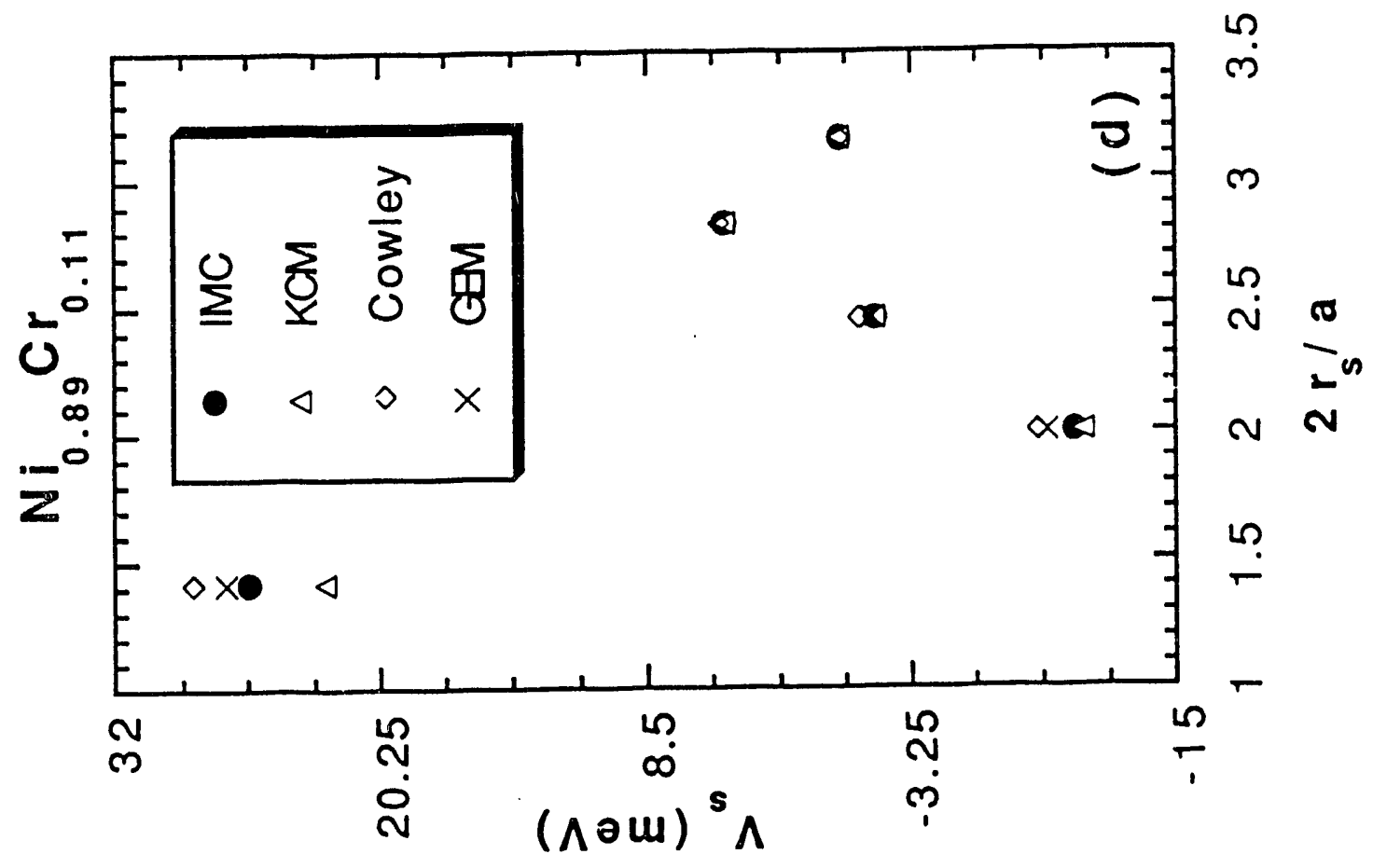



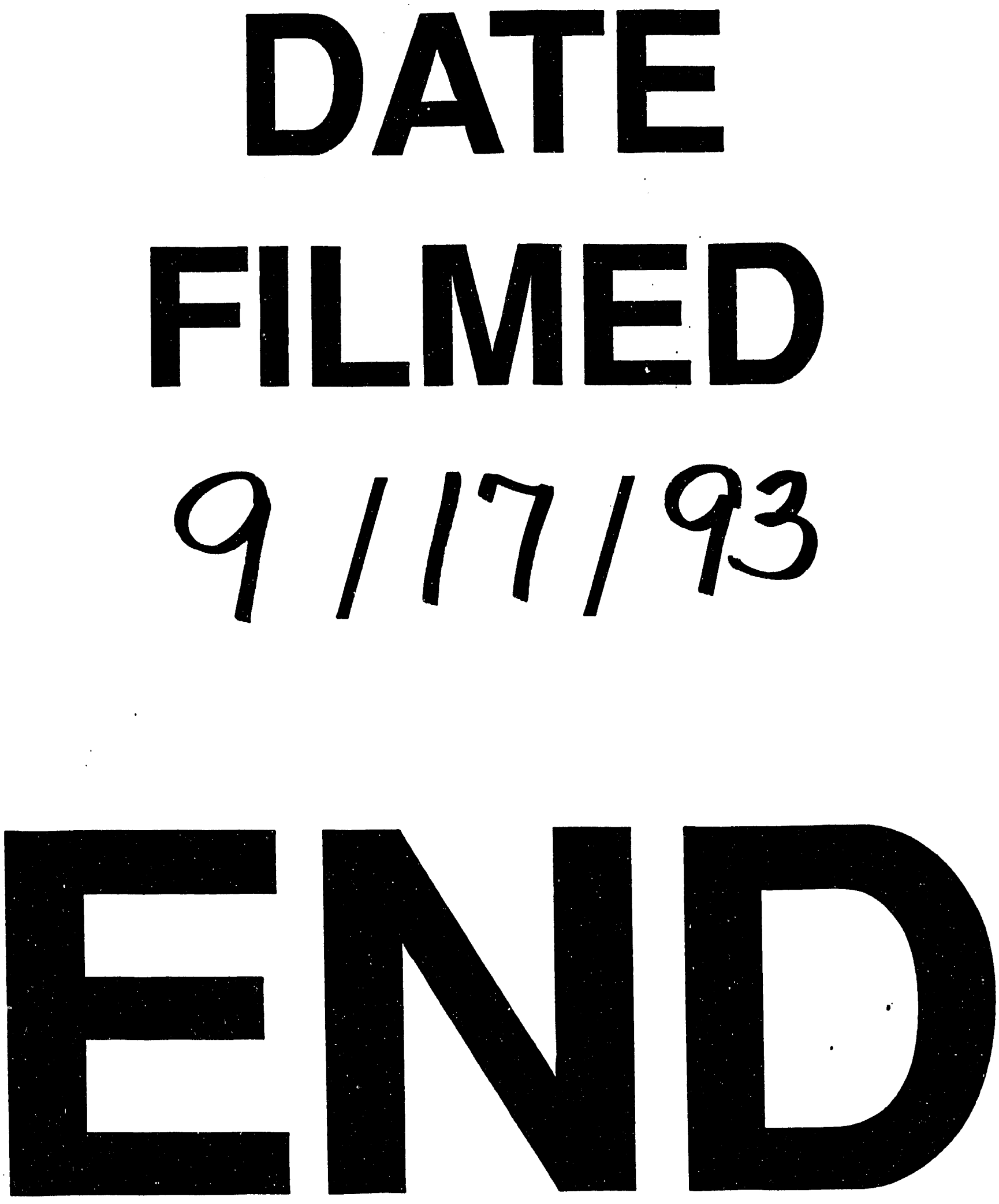


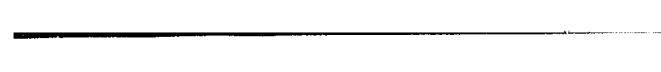

